\section{$\underset{\substack{\text { hommes } \\ \text { \& migrations }}}{ }$}

\section{Hommes \& migrations}

Revue française de référence sur les dynamiques

migratoires

$1305 \mid 2014$

L'exil chilien en France

\title{
Ces Français d'origine vietnamienne qui choisissent le retour
}

\author{
Laetitia Van Eeckhout
}

\section{OpenEdition}

\section{Journals}

Édition électronique

URL : http://journals.openedition.org/hommesmigrations/2761

DOI : 10.4000/hommesmigrations.2761

ISSN : 2262-3353

Éditeur

Musée national de l'histoire de l'immigration

Édition imprimée

Date de publication : 1 janvier 2014

Pagination : 164-167

ISBN : 978-2919-040261

ISSN : $1142-852 X$

\section{Référence électronique}

Laetitia Van Eeckhout, « Ces Français d'origine vietnamienne qui choisissent le retour », Hommes \& migrations [En ligne], 1305 | 2014, mis en ligne le 25 juillet 2014, consulté le 22 septembre 2020. URL : http://journals.openedition.org/hommesmigrations/2761 ; DOI : https://doi.org/10.4000/ hommesmigrations.2761 


\title{
REPÉRAGE
}

\section{CES FRANÇAIS D'ORIGINE VIETNAMIENNE QUI CHOISISSENT LE RETOUR}

\author{
LAETITIA VAN EECKHOUT, journaliste, auteur de France plurielle, \\ le défi de l'égalité réelle (Gallimard, "Folio Actuel", 2013).
}

Lrent e plus dur a été de faire admettre à ses parents qu'il ne commettait pas une folie. "Fais attention à toi et regarde où tu mets les pieds", lui a dit son père avant qu'il parte travailler au Vietnam, pays que sa famille avait fui trente-six ans plus tôt.

Dao, aujourd'hui quadragénaire, fait partie de ces Viêt-Kîeu, ces Vietnamiens d'outre-mer, comme les appellent ceux restés au pays. L'histoire de leurs familles a souvent été douloureuse. Certaines ont émigré en France à la chute de l'empereur Bao Dai en 1955.

D'autres sont arrivées à la chute de Saigon qui a signé la fin de la guerre du Vietnam. Mais un nombre croissant décident aujourd'hui de faire le grand voyage, parfois initiatique, vers leur pays d'origine.

Parti en 1975 à l'âge de 3 ans, Dao ne s'en est pas moins promis, une fois lancé dans la vie professionnelle, de revenir un jour dans son pays d'origine pour y travailler, s'y investir. Peut-être parce qu'il n'avait guère d'autres souvenirs que ses toutes premières années insouciantes de maternelle chez les religieuses françaises de Saigon. Entré dans un grand groupe français, il jette son dévolu sur la direction internationale, ce qui, de fil en aiguille, l'amène, lorsque le Vietnam commence à s'ouvrir, à faire des voyages éclairs de quelques jours pour négocier une licence pour son entreprise. Pour finir, en 2011, à décrocher un poste à Hô Chi Minh-Ville, l'ex-Saigon de son enfance.

\section{Un choix assumé}

Pour Bui aussi, le Vietnam a toujours été, et peutêtre plus encore, un fil rouge. S'il a été "vraiment éduqué à la française", ce Brestois d'adoption, comme il aime à se présenter, parti du Vietnam en 1978 à l'âge de 12 ans, dit n'avoir "jamais cessé d'être vietnamien". Une fois son diplôme d'actuaire en poche, il se débrouille dans ses premiers emplois pour être de toutes les missions lui permettant de "retrouver le pays de son enfance". Avant de pouvoir enfin effectuer en 2009, à 43 ans, un vrai retour, en prenant la direction de la succursale d'une compagnie d'assurances à Hô Chi Minh-Ville. Un vrai retour définitif. Car Bui, envoyé comme expatrié par son groupe, sait qu'il poursuivra, quoi qu'il arrive, sa carrière au Vietnam et y finira sa vie. "Très marqués par la guerre du Vietnam, mes parents resteront jusqu'à leur mort à Brest, en France. Mais moi, c'est plus fort que moi, c'est presque instinctif', dit-il.

Le retour de Duong ne fut, lui, pas instinctif, loin s'en faut. Ayant fui le Vietnam avec sa famille en 1951 - son père était secrétaire particulier de l'impératrice -, jamais il n'avait imaginé revenir vivre au pays. Jusqu'au jour où, quarante-deux ans plus tard, profitant d'un stage de sa fille étudiante en agronomie et spécialisée dans la culture du riz, il s'y est rendu "par curiosité", avec sa femme normande pour faire du tourisme. Et là ce fut le 
déclic. "Avec mes frères et sœurs, nous avons été élevés comme des Français. Je suis le seul à avoir entretenu le vietnamien de notre enfance. Le Vietnam n'était pas cependant mon pays. Mais quand je suis revenu en 1993, une fois sur place, j'ai dit à ma femme: 'Je me sens chez moi ici', racontet-il. Après ce voyage-là, a commencé à naître en lui l'idée de revenir vivre au Vietnam, d'autant que le groupe pour lequel il travaillait depuis vingt-huit ans scrutait alors ce marché promis à la croissance. Un projet qui se concrétise trois ans plus tard. Duong finit sa carrière au Vietnam et décide même d'y rester au moment de prendre sa retraite, pour ouvrir une librairie française, alors que ses trois enfants vivent à Paris.

\section{Quête de sens}

Bien que fortement ancrés dans la société française pour y être nés et y avoir grandi, des enfants décident de faire le chemin inverse de leurs parents. C'est une quête de sens qui a convaincu Duc de faire le grand saut, après avoir confortablement exercé pendant sept ans ses talents d'ingénieur dans deux entreprises de télécommunication. "Je versais plusieurs centaines d'euros à une association franco-vietnamienne. Mais faire un chèque sur un coin de table, c'est facile. Je finissais par me demander ce que je pouvais vraiment donner", explique ce quadragénaire qui, né à Paris dans le XIX arrondissement, a fait toute sa scolarité dans la banlieue Sud de Paris, avant d'intégrer l'École centrale de Paris. « Je suis culturellement français mais j'ai grandi dans une identité mixte. Et j'ai toujours eu envie de découvrir le pays de mes parents, et surtout de permettre à mes enfants de s'imprégner d'une culture que je ne saurais leur transmettre", livre-t-il. En 2005, avec un ami, Français lui aussi d'origine vietnamienne, Duc crée à Hô Chi Minh-Ville une société spécialisée dans l'externalisation, qui compte aujourd'hui déjà plus de 250 salariés : "Nous diversifions désormais notre portefeuille de clients. Mais à l'origine, nos clients sont des sociétés françaises, ce qui me permet de garder un lien avec mon pays." Mon pays... Tu-Tho a elle éprouvé le besoin de pleinement comprendre toutes les facettes de son identité, en venant s'installer au Vietnam. Un retour aux sources qui n'a pas toujours 


\section{REPÉRAGE}

sonné comme une évidence pour cette bouillonnante jeune femme de 26 ans, dont le père était arrivé en France en 1968 pour y faire ses études et la mère, d'une famille française d'Indochine, en 1975. "Avec ma soeur cadette, nous avons été élevées dans une double culture: nous parlions français, mangions souvent vietnamien, fêtions Noël comme le Têt. Pendant toute mon enfance et adolescence jusqu'à la fin de mes études, dit-elle, je ne me suis jamais sentie déchirée, ni même différente des autres." Jusqu'au moment d'entamer sa carrière où elle s'est vue brutalement interpellée sur son identité. Diplômée de Centrale Lille, elle était pourtant promise à un bel avenir. Mais lors des entretiens groupés organisés par l'école avec de grandes entreprises, aucun poste ne lui fut jamais proposé. La seule fois où elle décrocha un entretien, elle s'est entendu dire: "Si je vous prends aujourd'hui, dans deux ans vous serez à mon poste." Un a priori sur les Asiatiques qu'elle n'avait jamais imaginé se voir opposer. "Ce fut violent, se souvient Tu-Tho. J'ai alors voulu comprendre pourquoi on me renvoyait cette image d'étrangère, je me suis demandé s'il me manquait une part de mon identité."

"Se sentir pleinement française mais ne pas être considérée comme telle, à une période de sa vie où l'on se cherche, c'est difficile", explique Aurélie, qui garde elle aussi en mémoire le jour où l'un de ses professeurs lui a lâché : "Je suis très déçue par toi car tu devrais être première de la classe." Depuis ce jour-là, elle qui ne voulait "surtout pas être différente" s'est mise à s'interroger sur ses origines, et son envie de faire de l'international s'est muée en "besoin d'aller au Vietnam". Aujourd'hui, les deux jeunes femmes se disent pleinement épanouies: la première dirige un des restaurants les plus prisés d'Hô Chi Minh-Ville et une société de création d'événements ; la seconde est responsable du bureau de représentation d'une grande entreprise française au Vietnam, un poste qu'elle a décroché une fois sur place.

\section{Ce Vietnam dont ils aiment la vitalité}

En dépit de racines françaises assumées, tous apprécient l'allant, l'enthousiasme, la vitalité de la société vietnamienne, la manière dont les gens sont tournés vers l'avenir. Ils aiment l'esprit qui souffle de ce côté-là de la planète. Le Vietnam, qu'ils veulent aussi le leur, les fascine. Leurs débuts ne furent toutefois pas sans un certain désenchantement souvent. Le plongeon dans une culture qui n'est pas ou plus la sienne est loin d'être simple. Bien que très "excité" à l'idée de partir vivre au Vietnam, Dao reconnaît que "les six premiers mois ont été très rudes". "Je ne m'attendais pas à ce que la société vietnamienne soit aussi dure. Le Vietnam n'était pas du tout à l'image que je m'en faisais. Je n'avais jamais imaginé les fractures sociales, la dichotomie des gens", raconte-t-il. Ils pensent trouver ou retrouver une société avec laquelle ils partagent des références. Mais ils n'ont pas partagé avec elle tout un pan d'histoire qui a profondément marqué son peuple. "Même quand tu es intégrée, les Vietnamiens sont très fermés", témoigne Aurélie. "Il est très difficile de se sentir proche de Vietnamiens qui n'ont pas un temps vécu à l'étranger. Ily a un grand problème d'esprit critique. Le décalage est encore grand", avoue pour sa part Tu-Tho.

Viêt-Kîeu, Vietnamiens de l'étranger, ils ne sont plus considérés comme des "traîtres". Le régime communiste s'est assoupli à leur égard, en même temps que le pays optait pour l'ouverture de son économie. Pour autant, il n'est pas facile d'abattre le mur de méfiance qui leur est, au départ du moins, opposé. Même Bui, qui a passé toute son enfance au Vietnam, dit après cing ans ne pas être tout à fait vu comme un Vietnamien. "Il y a un certain complexe de leur part vis-à-vis de ceux qui ont été formés à l'étranger", explique-t-il. Tout 
jeune quadragénaire, Dan, dont les parents partis dans les années 1960 pour faire leurs études ne sont jamais revenus, ne se fait pas d'illusions. Entrepreneur dans l'âme ayant débarqué au Vietnam en 2008, après avoir grandi et, fait ses études en France, et débuté sa carrière aux ÉtatsUnis, il sait qu'il ne sera jamais perçu comme un Vietnamien. "Je suis un Français qui simplement, dit-il, arrive mieux que les autres à comprendre les Vietnamiens, acceptent plus facilement qu'ils fonctionnent différemment."

\section{Double culture sans déchirement}

Possédant malgré tout, de par leur histoire familiale, quelques codes de la société vietnamienne, ils ont "plus de facilités [que d'autres] à briser la glace". Et ils finissent par se découvrir une vraie double culture. "En France, je suis perçue comme une Vietnamienne, au Vietnam, comme une Française. Mais moi, je me sens pleinement francovietnamienne aujourd'hui", dit Tu-Tho. "La dualité est de plus en plus forte, reconnaît Duong. Mais, insiste-t-il, il n'y a pas de déchirement." II n'y a pas en eux de fracture qui les rendrait schizophrènes, avec chaque partie antagoniste de l'autre. Pleine dimension de leur identité, cette double culture leur apporte au contraire un plus, leur permet de s'adapter à des situations de forts contrastes, en ayant finalement face à celles-ci un certain détachement. Se voient-ils finir leurs jours au Vietnam ? Rares sont ceux qui sont catégoriques. Nul ne sait en fait ce que sera le Vietnam dans cinq ou dix ans. Face à cela, ils ont l'attitude bouddhiste de celui qui prend la vie telle qu'elle vient. En tout cas, aucun de ces Viêt-Kîieu n'a coupé ni même n'a envie de couper les ponts avec la France. "Le pays avec lequel on partage le plus de valeurs reste la France", dit Tu-Thau. Même Bui, qui n'imagine pas finir sa vie ailleurs qu'au Vietnam, aime retrouver sa Bretagne d'adoption.

\section{Une France trop peu présente}

La France, à laquelle ils restent attachés, ils l'aimeraient plus présente, plus active au Vietnam. Dans ce pays où plus de la moitié de la population a moins de 30 ans, la France, constatent-ils, appartient aux souvenirs des plus anciens, plus qu'elle n'est vivante dans le Vietnam contemporain. "Les Vietnamiens, relève Duong, savent qu'ily a un lien historique entre les deux pays, mais ils ne regardent pas la France comme porteuse d'avenir." "Pour les Vietnamiens, la France, c'est le luxe. Mais sans le luxe, elle ne serait rien aujourd'hui à leurs yeux", appuie Dao Nguyen qui constate amèrement que "la France n'a pas su mettre à profit son histoire avec le Vietnam pour y maintenir des liens, pour y rester vivante". II existe bien des entreprises françaises au Vietnam, mais c'est surtout dans une perspective de délocalisation qu'elles sont venues s'y implanter, intéressées avant tout par une main-d'œuvre bon marché. "Moins pour pleinement participer au développement du pays, jouer le partenariat avec les acteurs locaux, se tourner vers le consommateur vietnamien", regrette Paul Tran, boat-people ayant fui le pays en 1976, et revenu depuis déjà seize ans. Certes, les Vietnamiens connaissent La Vache qui rit. Certes, pas moins de 500 mots dans leur langue sont issus du français. "Mais ce ne sont là que des traces, dont la plupart des Vietnamiens ignorent l'origine française, relève Bui. La France n'investit pas assez, n'est pas assez investie dans le Vietnam d'aujourd'hui."Une chose est sûre, leur motivation première à eux n'est pas de faire fortune mais d'accompagner le Vietnam dans son développement. Et de voir les Vietnamiens ouvrir les yeux sur le monde, s'ouvrir au monde. Tous l'expriment à leur façon : pont entre deux cultures, ils veulent apporter leur contribution à cette ouverture. 